\title{
Métodos de análise da composição corporal em adultos obesos
}

\author{
Methods for body composition \\ analysis in obese adults
}

Rávila Graziany Machado de SOUZA'

Aline Corado GOMES'

Carla Marques Maia do PRADO²

João Felipe MOTA'

RE S U M O

Os métodos de avaliação da composição corporal em obesos têm sido amplamente discutidos, uma vez que nesses indivíduos a avaliação é dificultada devido às limitações dos equipamentos e características dos métodos utilizados. Esta sessão temática tem o objetivo de esclarecer as características, vantagens e limitações dos métodos de avaliação da composição corporal em adultos obesos. A quantificação de gordura corporal e massa livre de gordura, assim como a avaliação da perda de massa muscular e de massa óssea em obesos são temas de grande interesse científico, uma vez que são utilizados para diagnosticar a obesidade osteosarcopênica. A avaliação da composição corporal de obesos pelo modelo de múltiplos compartimentos é padrão-ouro na prática científica. Por outro lado, o método de absorciometria radiológica de feixe duplo é considerado o padrão de referência em pesquisas e na prática clínica. Estudos indicam que a ressonância magnética e a tomografia computadorizada, em alguns casos, são fortemente correlacionadas com a absorciometria radiológica de feixe duplo. Os demais métodos apresentam limitações em avaliar a composição corporal, bem como suas modificações durante a redução ponderal em indivíduos obesos.

Termos de indexação: Obesidade. Osteoporose. Sarcopenia.

A B S T R A C T

The analysis of body composition in obese individuals has been intensively discussed due to the methodological limitations of most measurement methods. The aim of this thematic section was to clarify the characteristics, advantages, and limitations of methods of body composition evaluation in obese adults. In addition to the

${ }^{1}$ Universidade Federal de Goiás, Faculdade de Nutrição, Laboratório de Investigação em Nutrição Clínica e Esportiva. R. 227, Qd. 68, s/n., Setor Leste Universitário, 74.605-080, Goiânia, GO, Brasil. Correspondência para/Correspondence to: JF

Mota.E-mail: <jfemota@gmail.com>.

2 University of Alberta, Chair in Nutrition Food and Health. Edmonton, AB, Canada.

Apoio: Fundação de Amparo à Pesquisa do Estado de Goiás (Processo no 201210267001125). 
measurement of body fat and fat-free mass, evaluation of specific patterns of body composition change, such as loss of both muscle and bone mass in obese individuals, is also of special scientific interest because they are used for the diagnosis and prognosis of osteosarcopenic obesity. Although the use of multi-compartment models are gold-standard for the assessment of body composition, dual-energy X-ray absorptiometry may be used as a method of reference in scientific research and clinical practice. Studies indicate that magnetic resonance and computed tomography are, in some instances, strongly correlated with dual-energy X-ray absorptiometry. Other techniques present with limitations in assessing the body composition of obese individuals, especially when evaluating weight loss changes.

Indexing terms: Obesity. Osteoporosis. Sarcopenia.

\section{N T R O D U ÇÃ O}

Nas últimas décadas, a obesidade tem crescido de forma vertiginosa em todo o mundo. No Brasil, o excesso de peso atinge $51 \%$ da população, e a obesidade representa $17 \%$ dos casos $^{1}$. Em países em desenvolvimento, o número de adultos acima do peso subiu quatro vezes nas últimas três décadas ${ }^{2}$, e nos Estados Unidos a prevalência de obesidade é de 34\% entre os homens e $55 \%$ entre as mulheres com idade entre 20 e 64 anos $^{2}$. Dessa forma, o diagnóstico precoce da obesidade é fundamental para a promoção da saúde e a redução da morbimortalidade, visto que a obesidade é fator de risco para outras doenças, além de reduzir a qualidade de vida ${ }^{3}$.

Do ponto de vista da composição corporal, a obesidade é caracterizada pela elevada quantidade de massa gorda e de água total extracelular ${ }^{4}$. Embora a medida da massa corporal total e o Índice de Massa Corporal (IMC) sejam índices conhecidos e utilizados na avaliação nutricional, a maioria das pesquisas concorda que eles são bastante imprecisos. A classificação de gordura de acordo com o IMC superestima a gordura corporal em indivíduos ativos e subestima a gordura corporal em indivíduos sedentários, obesos, idosos e em certas condições clínicas ${ }^{5-7}$.

A avaliação da composição corporal em obesos pode ser dificultada devido às limitações dos equipamentos utilizados e características dos métodos $^{8}$. Dessa forma, esta sessão temática tem o objetivo de esclarecer as características, vantagens e limitações dos métodos de avaliação da composição corporal em adultos obesos.

\section{Análise da massa adiposa em obesos}

A avaliação do percentual de gordura por dobras cutâneas é uma técnica duplamente indireta de avaliação da composição corporal ${ }^{9}$. Apesar de apresentar um menor custo financeiro e ser amplamente utilizada para a avaliação da composição corporal, essa técnica não é aconselhável para aferir o grau de adiposidade em indivíduos obesos, em razão de a gordura subcutânea não ser facilmente separada do músculo e também devido à limitação máxima de abertura do adipômetro. Isso compromete a exatidão da medida e subestima o total de gordura corporal ${ }^{7,10}$. A precisão das medidas das dobras cutâneas também é afetada por outros fatores, como a habilidade do avaliador, o tipo de compasso utilizado, a compressibilidade do tecido adiposo e o nível de hidratação do paciente ${ }^{11}$.

Em estudo com 78 pares de adultos gêmeos de ambos os sexos, foi avaliada a variação da gordura corporal total por meio de análise multivariada entre diferentes métodos. Observou-se que as estimativas de gordura corporal medida por Dual-Ernegy X-ray Absorptiometry (DEXA, Absorciometria de raio-X de Dupla Energia) e água corporal total não foram diferentes entre si. No entanto, foram maiores do que as estimativas de gordura corporal medidas pela Bioelectrial Impedance Analysis (BIA, Impedância Bioelétrica), dobras cutâneas e IMC, e menor do que a estimativa de gordura corporal medida por pesagem hidrostática. A precisão inferior da pesagem hidrostática foi atribuída à variação do teor de água e da densidade mineral óssea, e a subesti- 
mação do percentual de gordura pelas dobras cutâneas foi atribuída à habilidade do avaliador e ao tamanho das dobras cutâneas. Embora a medida da espessura das dobras cutâneas e a BIA sejam técnicas amplamente utilizadas devido à sua simplicidade e baixo custo, neste estudo elas subestimaram em $12 \%(p<0,001)$ a massa adiposa corporal total, quando comparada à medida pelo DEXA ${ }^{12}$. O coeficiente de variação da medida da composição corporal por meio de dobras cutâneas apresentou coeficiente de variação de $5 \%$, contra $2 \%$ do método DEXA ${ }^{13}$.

A capacidade de predição das equações também é um fator limitante para a utilização da medida das dobras cutâneas em determinadas populações. A comparação do percentual de gordura corporal avaliado por meio de dobras cutâneas e DEXA entre indivíduos eutróficos e obesos identificou uma inconsistência entre os resultados obtidos pelas diferentes equações utilizadas, apresentando resultados significativamente inferiores aos obtidos pelo DEXA, principalmente entre os indivíduos mais velhos e obesos ${ }^{14}$.

A Bioelectrial Impedance Analysis é um método rápido e não invasivo que avalia a composição corporal a partir da passagem de uma corrente elétrica pelo corpo do indivíduo, de modo que a resistência à passagem dessa corrente é mensurada ${ }^{15}$. A precisão da BIA pode ser afetada pelo equipamento utilizado, alimentação, ingestão hídrica, atividade física, ciclo menstrual e consumo de álcool ${ }^{16}$.

Estudo com indivíduos com sobrepeso e obesidade observou que a BIA, em conjunto com a circunferência da cintura apresentou melhor associação com a gordura corporal avaliada pela técnica DEXA do que o IMC ${ }^{17}$. Com relação à avaliação de modificações da composição corporal, um estudo observou que a impedância bioelétrica tetrapolar apresentou acurácia para detectar diferenças na composição corporal de indivíduos com sobrepeso em programa de perda de peso, apresentando coeficientes de correlação elevados com o DEXA (R percentual de gordura corpo- ral $=0,91, p=0,00 ; R$ massa livre de gordura $=0,92$, $p=0,00 ; R$ massa gorda $=0,99, p=0,00)^{18}$.

Apesar de a Bioelectrial Impedance Analysis com oito eletrodos ter sido validada utilizando o método pletismografia como referência, a população utilizada no estudo foi de homens e mulheres saudáveis ${ }^{19}$. Em mulheres obesas com idade entre 20 a 45 anos, a metodologia da BIA com oito eletrodos subestimou a massa adiposa de forma diretamente proporcional ao aumento da massa adiposa do avaliado ${ }^{20}$. As diferentes precisões observadas em estudos utilizando BIA com oito eletrodos se devem aos diferentes métodos de referência comparados e, também, às diferentes características das populações avaliadas (sexo, idade, IMC). Além disso, as diferentes taxas de hidratação observadas em obesos pode ser uma fonte de viés no método de BIA ${ }^{20}$. A hidratação pode ser influenciada pela ingestão dietética, consumo de álcool, nível de atividade física e fatores ambientais como umidade e temperatura ${ }^{21}$. A ingestão de cafeína e álcool podem causar desidratação e subestimar os valores de massa muscular em até cinco quilos. A prática de exercícios físicos de baixa intensidade não altera os valores; todavia, exercícios de moderada a alta intensidade podem superestimar a quantidade de massa muscular em até $12,0 \mathrm{~kg}$. A temperatura ambiente de $14^{\circ} \mathrm{C}$, comparada a de $35^{\circ} \mathrm{C}$, aumenta a resistência, reduzindo os valores de massa muscular em até $2,2 \mathrm{~kg}$. Outro ponto importante a ser considerado é que os resultados obtidos pela BIA e dobras cutâneas precisam ser inseridos em fórmulas preditoras, as quais não são adequadas para todas as faixas etárias e condições clínicas ${ }^{22}$.

Estudo que avaliou a composição corporal de 882 indivíduos de 18 a 88 anos observou que a BIA com oito eletrodos superestimou a massa magra e subestimou a massa gorda em homens (variação de 2,90\% da massa magra e de 15,85\% da massa gorda, $p<0,001$ ) e mulheres (variação de $7,30 \%$ da massa magra e de $16,14 \%$ da massa gorda, $p<0,001)$ quando comparada ao DEXA ${ }^{23}$. Em mulheres obesas participantes de um programa de perda de peso, a BIA apresentou elevada 
acurácia para avaliar a variação de massa adiposa e massa livre de gordura, quando utilizada como padrão de referência o método DEXA. Entretanto, em valores absolutos, a BIA de frequência única subestimou a massa adiposa e o percentual de gordura, além de superestimar a massa livre de gordura. Nesse mesmo estudo, a BIA de multifrequência não apresentou variação significativa com relação às mesmas variáveis, o que indica maior precisão quando comparada à BIA de frequência única ${ }^{24}$. Esse resultado também foi observado em outros estudos ${ }^{18,25}$.

Em contrapartida, estudo realizado com homens obesos observou que a BIA de multifrequência subestimou o percentual de gordura em $26,41 \%(p<0,01)$ comparado ao DEXA ${ }^{26}$. Essas diferenças podem ser decorrentes dos diferentes padrões de composição corporal observados entre homens e mulheres. Durante o ganho de peso, os homens aumentam massa adiposa e massa livre de gordura em proporções semelhantes, enquanto as mulheres apresentam maior ganho de massa adiposa em relação à massa livre de gordura ${ }^{27}$. Além disso, as mulheres apresentam maior acúmulo de gordura subcutânea, enquanto os homens apresentam maior acúmulo de gordura abdominal $^{28}$. Dessa forma, as diferenças de sexo e idade das populações avaliadas pela BIA podem justificar as diferentes acurácias observadas.

Outras explicações para as avaliações imprecisas pelo método BIA em obesos é que obesos graves apresentam alteração nas propriedades de condução elétrica dos tecidos, o que pode alterar a penetração da corrente elétrica ${ }^{29}$. Além disso, a maior quantidade de água extracelular observada em indivíduos obesos favorece a superestimação da massa livre de gordura e a subestimação da massa gorda ${ }^{26}$. A vantagem da BIA com oito eletrodos, em comparação com a de quatro eletrodos, é a avaliação compartimentada, que possibilita a avaliação da gordura visceral ${ }^{30}$.

A técnica de DEXA é baseada na divisão do corpo em três níveis: mineral-ósseo, massa magra e massa gorda ${ }^{31}$. O raio-X do DEXA tem potência constante que gera dois picos de energia
(40 KeV e $70 \mathrm{KeV}$ ), possibilitando a aferição dos tecidos $^{32}$. Esse método é uma alternativa à pesagem hidrostática, visto ser seguro, relativamente rápido e requerer mínima cooperação dos indivíduos. A avaliação da composição corporal por DEXA sofre menor influência da quantidade de água corporal do que a pesagem hidrostática; entretanto, não é indicado para gestantes, lactantes, portadores de implantes metálicos, marca-passo, e indivíduos submetidos a procedimentos com iodo, bário ou isótopos em um período de sete dias anteriores à avaliação ${ }^{15}$.

O Dual-Ernegy X-ray Absorptiometry é considerado o padrão de referência para a avaliação da composição corporal em obesos ${ }^{33}$, com menor demanda de tempo e emissão de radiação comparada à Tomografia Computadorizada (TC), além de apresentar maior facilidade de acesso do que a TC e a Ressonância Magnética (RM) ${ }^{34}$. Entretanto, o DEXA apresenta resolução inferior e coeficiente de variação superior comparado à TC e RM. As imagens da RM são em 3D, enquanto as do DEXA apenas em 2D ${ }^{34}$. De acordo com um estudo transversal, o DEXA apresentou forte correlação $(r=0,74 ; p<0,01)$ com o tecido adiposo abdominal avaliado por RM em mulheres; todavia, em homens essa correlação foi apenas moderada $(r=0,46 ; p<0,05)^{35}$. Comparado à TC, o DEXA apresentou elevada acurácia para a quantificação do tecido adiposo visceral ${ }^{36}$ em homens $(r=0,88$; $p<0,001)$ e mulheres $\left(r=0,94 ; p<0,001^{37}\right.$. A precisão da medida de tecido adiposo visceral em mulheres foi confirmada pelo estudo de Rothney et al. $(2013)^{38}$, que observou um coeficiente de variação de 5,1\% $(p<0,05)$.

É importante se atentar às limitações técnicas do aparelho, uma vez que o scanner pode não apresentar tamanho suficiente para avaliar completamente indivíduos obesos, resultando em erros na estimativa do percentual de gordura corporal $^{39}$. A capacidade de suportar a massa corporal do indivíduo é variável de acordo com marca e modelo do equipamento ${ }^{40}$, podendo avaliar indivíduos com massa corporal de até 204 kg, como no iDEXA $\left(\mathrm{GE}^{\odot}\right)$ ou no Discovery QDR 
(Hologic ${ }^{\odot}$ ). Além disso, obesos são "largos", atenuando significativamente os raios-X. Assim, alguns fabricantes fornecem modos de varredura especiais e técnicas de análise para tais pacientes. Esses modos de varredura, em geral, possuem as mesmas configurações de tensão do tubo de raios- $X$, mas com maior radiação ou tempo de varredura mais lento para aumentar a emissão de raios- $X^{41}$.

Em obesos com dimensões superiores aos limites das bordas da cama do aparelho de DEXA, o protocolo do meio-scanner (hemiscan) pode ser utilizado. Nesse protocolo, o indivíduo é posicionado no centro da linha da cama para que um lado do corpo, geralmente o lado direito, seja completamente incluído no campo do scanner. O protocolo meio-scanner apresenta excelente acurácia $\left(r^{2} \geq 0,98\right)$, não diferindo significativamente da aferição do corpo total| ${ }^{41}$.

A pletismografia estima o volume corporal a partir do deslocamento do ar e, com base na determinação da densidade corporal, avalia o percentual de gordura corporal. Para determinar o deslocamento do ar é utilizado um equipamento construído com fibra de vidro, acoplado a um computador, que determina as variações no volume de ar e pressão, ajustando a variação pulmonar. Esse método é relativamente rápido, porém apresenta elevado custo operacional e capacidade de $250 \mathrm{~kg}^{42}$.

A pletismografia pode apresentar erro ao determinar a densidade da massa livre de gordura em obesos, uma vez que estes apresentam maior quantidade de água extracelular ${ }^{43}$. Além disso, o volume de gás torácico é superestimado em indivíduos com sobrepeso e obesidade, resultando no aumento de 0,4 kg de massa adiposa em indivíduos com excesso de peso ${ }^{44}$. Quando comparada com a pesagem hidrostática, a pletismografia foi eficaz na determinação da densidade corporal de indivíduos com sobrepeso (variação de $0,09 \%)^{45}$. Em obesos, a pletismografia foi capaz de medir pequenas alterações de composição corporal; no entanto, subestimou a massa livre de gordura em $1 \mathrm{~kg}(p<0,001)$ e superestimou a adiposidade também em $1 \mathrm{~kg}(p<0,001)$, uma vez que uma maior hidratação (4 litros de água) foi detectada como aumento de gordura corporal $(4,69 \%, p<0,001)^{46}$.

A acurácia da pletismografia em comparação ao DEXA foi analisada em mulheres com sobrepeso e obesidade, antes e após um programa de perda de peso com duração de 16 semanas. Os resultados mostraram que a pletismografia apresentou resultados inferiores de porcentagem de gordura corporal (variação de 9,92\%, $p<0,001$ ) e de massa adiposa (variação de $8,80 \%$, $p<0,001)$, além de valores superiores de massa livre de gordura (variação de $7,19 \%, p<0,05$ ) quando comparada ao DEXA ${ }^{44}$. Resultados semelhantes foram observados em outro estudo com homens e mulheres com sobrepeso, após um programa de oito semanas de perda de peso ${ }^{47}$. Em contrapartida, Frisard et al. ${ }^{18}$ observaram resultado oposto em estudo também realizado com homens e mulheres com sobrepeso, participantes de um programa de perda de peso, em que a pletismografia apresentou resultados superiores de percentual de gordura corporal (variação de $12,14 \%, p=0,000$ ) e massa adiposa (variação de $11,02 \%, p=0,000)$, e inferiores de massa livre de gordura (variação de $9,70 \%, p=0,000$ ) quando comparada ao $\mathrm{DEXA}^{18}$. De acordo com estudo de Hames et al. ${ }^{48}$, a pletismografia superestimou a massa livre de gordura e subestimou a massa gorda e o percentual de gordura corporal em indivíduos eutróficos e com sobrepeso quando comparada ao DEXA, enquanto nos indivíduos obesos subestimou a massa livre de gordura e superestimou a massa gorda e o percentual de gordura corporal.

A pesagem hidrostática considera que o corpo é constituído por massa gorda e massa livre de gordura ${ }^{49}$. A partir da determinação da densidade corporal (peso no ar/peso na água), é possível estimar o percentual de gordura corporal. Esse método é preciso para a medição da densidade corporal, mas fatores como estágio do ciclo menstrual, horário do dia, atividade física e uso de medicamentos podem alterar o peso na água. 
Além disso, essa pesagem é relativamente demorada e requer adaptação ao meio líquido ${ }^{32}$.

Estudo realizado com obesos graves observou que o percentual de gordura analisado pela metodologia de pesagem hidrostática com e sem a cabeça submersa não diferiu entre os homens, mas apresentou diferença entre as mulheres, com resultado inferior quando a cabeça não estava submersa, numa variação de 5,6\% $(p<0,0001)^{50}$. Outro estudo com obesos graves observou como causas de exclusão para a realização da pesagem hidrostática a expressão de desconforto e/ou apreensão em se manter no tanque de água e a incapacidade de executar as manobras necessárias para o teste satisfatório, além de outras limitações físicas dos sujeitos ${ }^{8}$.

Na ressonância magnética, quando a energia da frequência de rádio é aplicada perpendicularmente à direção do campo magnético, os núcleos absorvem a energia e modificam seu alinhamento, liberando a energia armazenada, o que possibilita medir o número de núcleos de hidrogênio do tecido ${ }^{51}$. A repetição desse processo forma uma imagem de corte transversal e quantifica o tecido adiposo total e subcutâneo ${ }^{52}$. A RM apresenta boa correlação com a pesagem hidrostática. Apesar da vantagem de não utilizar radiação iônica, a medição é de custo elevado e apresenta dificuldade técnica ${ }^{49}$.

Estudos de Gallagher et al. ${ }^{53}$ e Bosaeus et al. ${ }^{20}$ realizados com obesos observaram que $\mathrm{o}$ método de RM subestimou a massa gorda (mulheres: $1,90 \mathrm{~kg}$ e homens: $5,68 \mathrm{~kg}, p=0,0001) \mathrm{e}$ $( \pm 2,00 \mathrm{~kg}, p<0,001)$, quando a pletismografia foi utilizada como método de referência. Esse resultado foi neutralizado quando analisada uma população composta por obesos e eutróficos; entretanto, a análise individual por essa metodologia não apresentou acurácia suficiente ${ }^{20}$. Comparando-se RM com DEXA, estudo com obesos participantes de um programa de perda de peso observou que o DEXA subestimou a massa gorda (variação de $3,1 \mathrm{~kg}, p<0,05$ ) e que essa subestimação foi diretamente proporcional à gordura corporal dos indivíduos. No mesmo estudo, o DEXA superestimou a perda de gordura corporal, mas apresentou correlação significativa em relação à medida de massa magra comparada ao método de $\mathrm{RM}^{54}$.

A interactância de raios infravermelhos supõe que as medidas de interactância podem estimar a composição corporal a partir da densidade óptica, que é inversamente proporcional ao percentual de gordura corporal ${ }^{55}$. Para a realização dessa técnica utiliza-se um espectofotômetro de raios infravermelhos ${ }^{13}$. A acurácia pode ser modificada pelos instrumentos utilizados, prática do avaliador e distribuição da gordura corporal do avaliado $^{15}$.

Após a comparação dos dados de composição corporal do DEXA com os da interactância de raios infravermelhos em mulheres obesas, concluiu-se que esse método não deve ser utilizado de forma isolada para avaliar a composição corporal em pacientes obesos, seja na pesquisa científica ou na prática clínica, uma vez que foram observadas diferenças significativas entre dados relativos de gordura e massa magra ${ }^{56}$.

A Ultrassonografia (US) funciona a partir da conversão de energia elétrica em ondas sonoras, que passam através do tecido adiposo, muscular e ósseo, de modo que parte da energia é refletida no receptor da sonda e transformada em energia elétrica. Dessa forma, a US quantifica a espessura do tecido muscular e adiposo, apresentando boa correlação com o método de pesagem hidrostática ${ }^{52}$.

Já a tomografia computadorizada, a partir da atenuação do raio- $X$ nos diferentes tecidos, constrói uma imagem bidimensional em corte transversal do corpo ${ }^{52}$. As limitações desse método dizem respeito à exposição à radiação, principalmente em gestantes e crianças, ao alto custo do equipamento e à necessidade de técnicos especializados $^{13}$. Contudo, imagens de tomografia realizadas para diagnóstico e acompanhamento de pacientes podem ser utilizadas para avaliação da composição corporal ${ }^{57}$.

Estudo que comparou a distribuição de gordura corporal avaliada por US e TC em indi- 
víduos com obesidade grave observou elevada correlação entre a TC e US na determinação da gordura visceral $(r=0,95 ; p<0,05)$ e subcutânea $(r=0,72 ; p<0,05)^{58}$. Da mesma forma, a quantificação do tecido adiposo visceral pelo método US apresentou forte correlação $(r=0,64 ; p<0,05)$ com a medida da TC em mulheres obesas em pré-menopausa ${ }^{59}$. Em adultos com sobrepeso $(r=0,98 ; p<0,05)^{60}$, a US mostrou elevada acurácia para avaliar a gordura corporal comparada ao DEXA. Um resumo das características, vantagens e limitações desses métodos de avaliação da composição corporal em adultos obesos está disponível no Quadro 1.

\section{Análise da massa muscular em obesos}

A avaliação da composição corporal é comumente realizada pela quantificação da massa adiposa e da massa livre de gordura, sendo um parâmetro utilizado para avaliação de riscos metabólicos e cardiovasculares ${ }^{61}$. A avaliação da perda de massa muscular é de grande interesse científico, com foco na avaliação das características quantitativas e qualitativas do músculo esquelético. Os métodos de imagem e impedância bioelétrica permitem identificar valores preditivos de sarcopenia ${ }^{61}$.

A sarcopenia consiste na redução da capacidade funcional da massa muscular e da força física, com consequente redução da mobilidade, vitalidade, qualidade de vida e aptidão cardiorrespiratória ${ }^{5}$.

A sarcopenia ocorre, muitas vezes, de forma concomitante ao aumento da massa gorda, sendo denominada obesidade sarcopênica. Obesos com IMC superior a $40 \mathrm{~kg} / \mathrm{m}^{2}$ são mais suscetíveis à redução de massa corporal magra e aumento progressivo da massa gorda. Nesses casos, a perda de massa muscular não resulta em mudança no IMC, levando ao quadro de obesidade sarcopênica ${ }^{61}$.

O interesse em definir a sarcopenia iniciou-se por uma analogia ao IMC para encontrar o índice relativo de massa esquelética ${ }^{62,63}$. Usando modelos alométricos baseados em correlações empíricas, Heymisfield et al. ${ }^{63}$ mostraram que a composição corporal avaliada pela inclusão do músculo esquelético dimensionava a altura com o poder igual ou superior a 2, para a maioria dos grupos, segundo sexo e raça, daí a razão da criação de uma altura ao quadrado variável ajustada $^{63}$. Assim, a sarcopenia é avaliada pela relação entre o Músculo Esquelético Apendicular (MEA) dividido pela altura ao quadrado $\left(\mathrm{MEA} / \mathrm{m}^{2}\right)$, definindo o índice relativo de massa muscular. Indivíduos com índice menor do que $7,26 \mathrm{~kg} / \mathrm{m}^{2}$ para homens e $5,45 \mathrm{~kg} / \mathrm{m}^{2}$ para mulheres são classificados como sarcopênicos ${ }^{64}$.

A sarcopenia pode ser avaliada pelos métodos BIA e DEXA. Na determinação por DEXA, a quantificação dos valores de gordura corporal e dos tecidos moles do esqueleto apendicular é obtida por um scanner de corpo inteiro. Na análise por BIA, o valor da estatura $(\mathrm{cm})$ ao quadrado pelo da resistência ( $\mathrm{cm} / \mathrm{R}^{2}$, ohms) são utilizados para a estimativa da massa muscular esquelética corporal, por meio do modelo de regressão proposto por Janssen et al. ${ }^{65}$, ajustado por sexo e idade. A limitação do diagnóstico de sarcopenia realizado pelo método BIA é o fato de que a relação do músculo esquelético corporal e massa corporal total é dependente da gordura corporal e da hidratação dos tecidos magros, podendo existir grande variabilidade entre essas medidas, conforme comentado anteriormente ${ }^{66}$.

A obesidade sarcopênica é a combinação da baixa reserva de massa magra e elevada reserva de massa adiposa. Foi definida inicialmente por valores inferiores a 2 desvios-padrão, comparados à população referência específica quanto ao gênero ou valores de percentual de gordura, superiores a $27 \%$ para homens e $38 \%$ para mulheres $^{62,67}$. A sarcopenia pode ser subestimada em obesos e idosos, uma vez que a combinação de baixa reserva de massa magra e maior reserva de gordura pode resultar em uma massa corporal normal. O DEXA é considerado o padrão-ouro para o diagnóstico da obesidade sarcopênica ${ }^{68}$. 
Quadro 1. Características, vantagens e limitações de alguns métodos de avaliação da composição corporal em adultos obesos.

\begin{tabular}{|c|c|c|c|c|}
\hline Técnicas & $\begin{array}{c}\text { Princípio e compartimentos } \\
\text { mensurados }\end{array}$ & Vantagens & Desvantagens & $\begin{array}{l}\text { Sugestão de } \\
\text { referências }\end{array}$ \\
\hline \multicolumn{5}{|l|}{ Antropometria } \\
\hline Dobras cutâneas & $\begin{array}{l}\text { Medida da espessura de } \\
\text { gordura representativa da } \\
\text { camada de gordura sub- } \\
\text { cutânea. }\end{array}$ & $\begin{array}{l}\text { Rápida, não invasi- } \\
\text { va, baixo custo e fa- } \\
\text { cilidade de trans- } \\
\text { porte de equipa- } \\
\text { mentos. }\end{array}$ & $\begin{array}{l}\text { Baixa precisão pela variação da medi- } \\
\text { da inter e intra-avaliadores; limitação } \\
\text { máxima de abertura do adipômetro; } \\
\text { subestimação da gordura corporal } \\
\text { total. Os resultados precisam ser in- } \\
\text { seridos em fórmulas preditoras, não } \\
\text { adequadas para todas as faixas etárias } \\
\text { e condições clínicas. }\end{array}$ & $\begin{array}{c}7,10,11 \\
14,22\end{array}$ \\
\hline \multicolumn{5}{|c|}{ Volume corporal/Densidade } \\
\hline Pesagem hidrostática & $\begin{array}{l}\text { Densidade corporal a par- } \\
\text { tir da razão peso no ar/ } \\
\text { peso na água. }\end{array}$ & Elevada precisão. & $\begin{array}{l}\text { Demorada, requer adaptação no meio } \\
\text { líquido, desconfortável para obesos, } \\
\text { precisão afetada por estágio do ciclo } \\
\text { menstrual, horário do dia, atividade } \\
\text { física e uso de medicamentos. }\end{array}$ & $8,13,32$ \\
\hline Pletismografia & $\begin{array}{l}\text { Volume corporal a partir } \\
\text { do deslocamento do ar. }\end{array}$ & $\begin{array}{l}\text { Relativamente rápi- } \\
\text { da, capacidade de } \\
250 \mathrm{~kg} \text {. }\end{array}$ & $\begin{array}{l}\text { Superestima o volume de gás torácico } \\
\text { em casos de sobrepeso/obesidade; } \\
\text { erro ao determinar densidade da MLG } \\
\text { em obesos; elevado custo. Não for- } \\
\text { nece dados de segmentos corporais. }\end{array}$ & $42,43,44$ \\
\hline \multicolumn{5}{|l|}{ Impedância } \\
\hline Impedância bioelétrica & $\begin{array}{l}\text { Passagem de uma corren- } \\
\text { te elétrica pelo corpo. A } \\
\text { impedância pode ser cal- } \\
\text { culada medindo-se a cor- } \\
\text { rente e a voltagem. }\end{array}$ & $\begin{array}{l}\text { Rápida, não } \\
\text { invasiva. }\end{array}$ & $\begin{array}{l}\text { Precisão afetada pelo equipamento } \\
\text { utilizado, alimentação, hidratação, } \\
\text { água extracelular, atividade física, } \\
\text { temperatura, ciclo menstrual e con- } \\
\text { sumo de álcool. Dados pouco preci- } \\
\text { sos para obesos graves, os resultados } \\
\text { precisam ser inseridos em fórmulas } \\
\text { preditoras, não adequadas para todas } \\
\text { as faixas etárias e condições clínicas. }\end{array}$ & $\begin{array}{l}15,16,20 \\
21,22,29\end{array}$ \\
\hline
\end{tabular}

Imagem/Atenuação por raios-X/Raios infravermelhos

\begin{tabular}{|c|c|c|c|}
\hline $\begin{array}{l}\text { Absorciometria radiológica } \\
\text { de feixe duplo }\end{array}$ & $\begin{array}{l}\text { Divisão do corpo em três } \\
\text { níveis: mineral-ósseo, MLG } \\
\text { e MG por meio de raios-X. }\end{array}$ & $\begin{array}{l}\text { Segura, relativa- } \\
\text { mente rápida, re- } \\
\text { quer mínima coo- } \\
\text { peração, sofre me- } \\
\text { nor influência da } \\
\text { quantidade de água } \\
\text { corporal do que a } \\
\text { pesagem hidrostá- } \\
\text { tica, padrão de re- } \\
\text { ferência para ava- } \\
\text { liação da composi- } \\
\text { ção corporal de obe- } \\
\text { sos, protocolo he- } \\
\text { miscan, rápida, } \\
\text { baixa emissão de ra- } \\
\text { diação. }\end{array}$ & $\begin{array}{l}\text { Limite de } 204 \mathrm{~kg} \text {, tamanho do scanner, } 15,31,32 \text {, } \\
\text { não é indicada para gestantes, lactan- } 33,34,40,41 \\
\text { tes, portadores de implantes metáli- } \\
\text { cos, marcapasso, nem para indiví- } \\
\text { duos submetidos a procedimentos } \\
\text { com iodo, bário ou isótopos em um } \\
\text { período de sete dias anteriores. Ima- } \\
\text { gens em 2D. }\end{array}$ \\
\hline
\end{tabular}


Quadro 1. Características, vantagens e limitações de alguns métodos de avaliação da composição corporal em adultos obesos.

\begin{tabular}{|c|c|c|c|c|}
\hline Técnicas & $\begin{array}{l}\text { Princípio e compartimentos } \\
\text { mensurados }\end{array}$ & Vantagens & Desvantagens & $\begin{array}{l}\text { Sugestão de } \\
\text { referências }\end{array}$ \\
\hline $\begin{array}{l}\text { Tomografia } \\
\text { computadorizada }\end{array}$ & $\begin{array}{l}\text { A partir atenuação dos } \\
\text { raios-X nos diferentes teci- } \\
\text { dos, fornece imagem bidi- } \\
\text { mensional em corte } \\
\text { transversal do corpo. }\end{array}$ & $\begin{array}{l}\text { Boa correlação } \\
\text { entre a circunfe- } \\
\text { rência da cintura } \\
\text { e o tecido adiposo } \\
\text { abdominal } \\
\text { profundo, } \\
\text { imagens 3D. }\end{array}$ & $\begin{array}{l}\text { Exposição à radiação, alto custo do } \\
\text { equipamento e necessidade de téc- } \\
\text { nicos especializados. }\end{array}$ & $13,34,52,57$ \\
\hline Ressonância magnética & $\begin{array}{l}\text { A partir da frequência de } \\
\text { rádio, é realizada a medi- } \\
\text { ção do número de núcle- } \\
\text { os de hidrogênio do teci- } \\
\text { do. Quantifica a MG total } \\
\text { e subcutânea. }\end{array}$ & $\begin{array}{l}\text { Apresenta boa cor- } \\
\text { relação com a pesa- } \\
\text { gem hidrostática, } \\
\text { não utiliza radiação } \\
\text { iônica, imagens 3D. }\end{array}$ & Custo elevado, dificuldade técnica. & $34,49,51,52$ \\
\hline Ultrassonografia & $\begin{array}{l}\text { Conversão de energia elé- } \\
\text { trica em ondas sonoras, } \\
\text { que passam através dos } \\
\text { tecidos, quantificando a } \\
\text { espessura dos tecidos } \\
\text { muscular e adiposo. }\end{array}$ & $\begin{array}{l}\text { Boa correlação com } \\
\text { o método de pesa- } \\
\text { gem hidrostática e } \\
\text { absorciometria radi- } \\
\text { ológica de feixe } \\
\text { duplo. }\end{array}$ & $\begin{array}{l}\text { Elevado custo e dificuldades } \\
\text { técnicas. }\end{array}$ & 52,60 \\
\hline $\begin{array}{l}\text { Interactância de raios } \\
\text { infravermelhos }\end{array}$ & $\begin{array}{l}\text { Supõe que as medidas de } \\
\text { interactância podem esti- } \\
\text { mar a composição corpo- } \\
\text { ral a partir da densidade } \\
\text { óptica, que é inversamen- } \\
\text { te proporcional ao percen- } \\
\text { tual de gordura corporal. } \\
\text { Modelo de múltiplos com- } \\
\text { partimentos. }\end{array}$ & $\begin{array}{l}\text { Rápida e não } \\
\text { invasiva. }\end{array}$ & $\begin{array}{l}\text { Acurácia modificada pelos instru- } \\
\text { mentos utilizados, prática do avalia- } \\
\text { dor e distribuição da gordura corpo- } \\
\text { ral do avaliado. } \\
\text { Não deve ser utilizada de forma iso- } \\
\text { lada para avaliar a composição cor- } \\
\text { poral em pacientes obesos. }\end{array}$ & $15,55,56$ \\
\hline \multicolumn{5}{|c|}{ Modelo de múltiplos compartimentos } \\
\hline $\begin{array}{l}\geq 3 \text { Modelos de } \\
\text { Compartimento }\end{array}$ & $\begin{array}{l}\text { Fracionamento antropo- } \\
\text { métrico da massa corpo- } \\
\text { ral utilizando combinação } \\
\text { de métodos. Como regra } \\
\text { geral para cada compo- } \\
\text { nente estimado, utiliza-se } \\
\text { uma equação que inclui o } \\
\text { componente desconheci- } \\
\text { do, o componente conhe- } \\
\text { cido e/ou o compartimen- } \\
\text { to mensurável. Compo- } \\
\text { nentes geralmente utiliza- } \\
\text { dos para o modelo de } \\
\text { quatro compartimentos: } \\
\text { água corporal total com } \\
\text { água deuterada ou água } \\
\text { enriquecida com } 180{ }_{2} \text {; } \\
\text { massa corporal; volume } \\
\text { corporal por plestimogra- } \\
\text { fia ou pesagem hidrostá- } \\
\text { tica; conteúdo mineral ós- } \\
\text { seo. }\end{array}$ & $\begin{array}{l}\text { Padrão ouro para } \\
\text { monitoramento de } \\
\text { mudanças na com- } \\
\text { posição corporal e } \\
\text { validação de outras } \\
\text { técnicas de compo- } \\
\text { sição corporal. }\end{array}$ & $\begin{array}{l}\text { Necessidade de mais equipamentos, } \\
\text { tempo e conhecimento técnico. }\end{array}$ & 6 \\
\hline
\end{tabular}

MG: Massa Gorda; MLG: Massa Livre de Gordura; IMC: Índice de Massa Corporal. 
No entanto, apesar do crescente interesse em compreender esse fenótipo de composição corporal, um consenso de diagnóstico ainda não foi alcançado, como é evidente nos resultados contrastantes relatados na literatura médica ${ }^{57}$. A prevalência de obesidade sarcopênica em adultos mais velhos pode variar até 26 vezes, dependendo das definições de valores baseados na literatura atual. Esse alto grau de variabilidade sugere a necessidade de se estabelecerem critérios de consenso que possam ser aplicados de forma confiável nas pesquisas clínicas ${ }^{69}$.

\section{Análise da massa óssea em obesos}

A osteoporose, problema de saúde pública, é caracterizada pela redução da massa óssea, afetando cerca de $50 \%$ das mulheres com idade igual ou superior a $50 \operatorname{anos}^{70}$. A avaliação da massa óssea em obesos muitas vezes é negligenciada. A obesidade parece moderar os efeitos da osteoporose, pelo aumento da densidade mineral óssea ${ }^{71}$. O aumento da massa corporal estimula o aumento da massa óssea; assim, apresentar um IMC elevado seria um fator de proteção para osteoporose ${ }^{70}$.

Ainda é desconhecido se a etnia é fator determinante, uma vez que a maioria dos estudos foi realizada com mulheres brancas ${ }^{70,72}$. Para avaliar a influência do excesso de massa corporal sobre a Densidade Mineral Óssea (DMO) em diferentes etnias, foi realizado um estudo com mulheres pós-menopausadas de três diferentes etnias, com idade média de 58 anos e IMC médio de $30,6 \mathrm{~kg} / \mathrm{m}^{2}$. A densidade mineral óssea da coluna lombar foi analisada por DEXA, considerando T-score menor que -1. As mulheres afro-americanas tiveram menor chance de ter baixa densidade mineral óssea quando comparadas às brancas [Odds ratio $(\mathrm{OR})=0.079(0.03-0.24)$ $(95 \% \mathrm{Cl}), p<0,01]$. No entanto, a DMO não apresentou diferenças significativas entre mulheres brancas e latino-americanas. Na análise por etnias, em mulheres brancas, a cada aumento de unidade de IMC, houve redução na chance de DMO
[OR=0.9 (0.87-0.94), $p<0,01]$. Em mulheres afro-americanas foi observada maior probabilidade de redução na DMO para cada aumento de uma unidade de IMC $[O R=1.015$ (1.007-1.14), $p<0,01]^{72}$.

Em estudo transversal com 4642 mulheres com idade entre 50 e 79 anos, foi verificada variação da $\mathrm{DMO}$ e geometria do fêmur de acordo com o IMC. A DMO do fêmur e a força geométrica foram maiores em mulheres com excesso de peso. Todavia, quando os dados foram corrigidos, essa associação estava relacionada principalmente à massa muscular, e não à massa corporal ou adiposa. Além disso, houve maior número de relatos de queda entre as obesas, as quais apresentaram menor força no fêmur. Apesar dos traumas, houve menor incidência de fraturas de quadril e outras áreas do corpo, o que os autores justificaram pela redução do impacto pela presença do tecido adiposo $^{73}$.

O Dual-Ernegy X-ray Absorptiometry é considerado padrão-ouro pela World Health Organization $^{74}$ para avaliação da osteoporose. A medição pode ser feita na parte inferior da coluna vertebral, fêmur e quadril. Trata-se de um método rápido, preciso e de baixa exposição à radiação, pelo qual podem ser detectadas perdas ósseas inferiores a $5 \%$, enquanto o método por raio- $X$ convencional detecta perdas ósseas a partir de $30 \%{ }^{75}$. Outra vantagem desse método é a possibilidade de comparar sítios com maior ou menor DMO com as respectivas reservas adiposas. Em um estudo com 2347 adultos, foi observada correlação negativa entre a DMO e a proporção de gordura em membros inferiores, e correlação positiva entre a gordura do tronco e a densidade mineral óssea em ambos os sexos ${ }^{76}$.

Outra desvantagem do exame radiológico convencional em obesos é a maior carga de radiação, com o propósito de garantir a correta interpretação dos resultados, uma vez que a maior espessura do obeso atenua a radiação e pode interferir na diferenciação da densidade entre os diferentes tecidos ${ }^{77}$. Por outro lado, a RM é um método não-invasivo e sem emissão de radiação 
também utilizado para examinar ossos, articulações e tecidos moles. Mas seu principal objetivo é avaliar a deterioração das articulações resultantes de artrite. Uma coorte com 192 pacientes obesos e idosos mostrou correlação positiva entre a RM e o exame radiológico tradicional para avaliação de osteoartrite ${ }^{78}$. A TC quantitativa também é um método que permite o diagnóstico da densidade mineral óssea volumétrica, porém o paciente é submetido a altas doses de radiação ${ }^{79}$.

Um novo conceito combinado para definir massa muscular, diminuição da força e massa óssea com a coexistência de hiperadiposidade é definido como obesidade osteopênica ${ }^{80,81}$. Esses autores sugerem que, sob determinadas condições, a osteoporose e a sarcopenia associadas à obesidade poderiam ser definidas como obesidade osteosarcopênica. Um estudo com 183 idosos neozelandeses mostrou que a osteoporose na região femoral mostrou incidência de $22 \%$ nos sarcopênicos, $17 \%$ nos obesos sarcopênicos, $12 \%$ nos eutróficos e $7 \%$ nos obesos. Além disso, a osteoporose nos sarcopênicos esteve relacionada a deficits de equilíbrio e marcha, especialmente nos obesos sarcopênicos ${ }^{82}$.

Em um recente estudo longitudinal "Geelong Osteoporosis Study" -, foi observado o aumento da massa adiposa, com diminuição de massa magra e óssea. Os autores sugerem que tais alterações implicam o desenvolvimento de fragilidade óssea, sarcopenia e obesidade sarcopênica ${ }^{83}$.

\section{O N CLUSÃ O}

A análise da composição corporal é imprescindível para o diagnóstico e conduta nutricional. A quantificação e diferenciação do tecido gorduroso e dos tecidos livres de gordura minimizam erros de interpretação para a tomada de decisões. A escolha do método para avaliação da composição corporal deve ser feita considerando as vantagens e limitações inerentes a cada uma das metodologias e da população que se pretende investigar.
Nesta revisão, foram apresentados os principais erros e limitações dos métodos para avaliação da composição corporal em obesos adultos, para os quais se deve levar em consideração não apenas a quantificação de gordura corporal, mas também a perda de massa muscular e de massa óssea, como forma de investigar possíveis casos de obesidade sarcopênica ou osteosarcopênica.

Atualmente, o método DEXA é considerado padrão de referência em pesquisas e na prática clínica para a avaliação da composição corporal em obesos, principalmente por se tratar de um método rápido e seguro. Ainda assim, apresenta limitação quanto ao tamanho do scanner do equipamento e à maior atenuação do raio- $X$, não sendo indicado em indivíduos com massa corporal superior a $204 \mathrm{~kg}$. Os métodos de RM e TC possuem correlação com o DEXA em algumas populações, mas são metodologias com maior custo e exposição. Dessa forma, deve-se investir em novos estudos, tanto para investigar correlações na quantificação dos diferentes componentes corporais em indivíduos obesos, quanto para o desenvolvimento de metodologias mais específicas para a avaliação da composição corporal nessa população.

\section{AGRADECIMENTOS}

Ao Conselho Nacional de Desenvolvimento Científico e Tecnológico, à Coordenação de Aperfeiçoamento de Pessoal de Nível Superior e à Fundação de Amparo à Pesquisa do Estado de Goiás, pelo apoio financeiro.

\section{COLABORADORES}

Todos os autores participaram de todas as fases da pesquisa do artigo.

\section{REFERÊ NCIAS}

1. Brasil. Ministério da Saúde. Vigitel Brasil 2012: vigilância de fatores de risco e proteção para doenças crônicas por inquérito telefônico. Brasília: Ministério 
da Saúde; 2012 [acesso 2014 jan 12]. Disponível em: <http://bvsms.saude.gov.br/bvs/publicacoes/ vigitel_2012.pdf>.

2. World Health Organization. World health statistics: Progress on the health-related millennium development goals. Geneva: WHO; 2012 [cited 2013 Nov 30]. Available from: <http://www. who.int/gho/publications/world_health_statistics/ 2012/en /index.html>.

3. Moyer VA. Screening for and management of obesity in adults: U.S. preventive services task force recommendation statement. Ann Intern Med. 2012; 157(5):1-32. doi: 10.7326/0003-4819-157-5-2 01209040-00482

4. Beechy L, Galpern J, Petrone A, Das SK. Assessment tools in obesity: Psychological measures, diet, activity, and body composition. Physiol Behav. 2012; 107(1):154-71. doi: 10.1016/j.physbeh.2012. 04.013

5. Prado CM, Wells JC, Smith SR, Stephan BC, Siervo M. Sarcopenic obesity: A critical appraisal of the current evidence. Clin Nutr. 2012; 31(5):583-601. doi: 10.1016/j.clnu.2012.06.010

6. Heymsfield SB, Wang Z, Baumgartner RN, Ross R. Human body composition: Advances in models and methods. Annu Rev Nutr. 1997; 17(1):527-58.

7. Lutoslawska G, Malara M, Tomaszewski P, Mazurek K, Czajkowska A, Kêska A, et al. Relationship between the percentage of body fat and surrogate indices of fatness in male and female Polish active and sedentary students. J Physiol Anthropol. 2014; 33(1):10. doi: 10.1186/1880-6805-33-10

8. Das SK. Body composition measurement in severe obesity. Curr Opin Clin Nutr Metab Care. 2005; 8(6):602-6.

9. Heyward V, Stolarczyk L. Applied body composition assessment. Champaign (IL): Human Kinetics Books; 1996.

10. Jackson AS, Pollock ML. Pratical assessment of body composition. Physician Sport Med. 1985; 13:256-62.

11 Martin AD, Drinkwater DT, Clarys JP, Daniel M, Ross WD. Effects of skin thickness and skinfold compressibility on skinfold thickness measurement. Am J Hum Biol. 1992; 4(4):453-60.

12. Elder SJ, Roberts SB, McCrory MA, Das SK, Fuss PJ, Pittas AG, et al. Effect of body composition methodology on heritability estimation of body fatness. Open Nutr J. 2012; 6(1):48-58. doi: $10.2174 / 1874288201206010048$

13. Lukaski HC. Methods for the assessment of human body composition: Traditional and new. Am J Clin Nutr. 1987; 46(4):537-56.

14. Chambers AJ, Parise E, McCrory JL, Cham R. A comparison of prediction equations for the estimation of body fat percentage in non-obese and obese older Caucasian adults in the United States. J Nutr Health Aging. 2014; 18(6):586-90. doi: 10.1007/s12603-014-0017-3

15. Heyward VH. Practical body composition assessment for children, adults, and older adults. Int J Sport Nutr. 1998; 8(3):285-307.

16. Heyward VH, Cook KL, Hicks VL, Jenkins KA, Quatrochi JA, Wilson WL. Predictive accuracy of three field methods for estimating relative body fatness of nonobese and obese women. Int J Sport Nutr. 1992; 2(1):75-86.

17. Di Renzo L, Rizzo M, Lacopino L, Sarlo F, Domino E, Jacoangeli F, et al. Body composition phenotype: Italian Mediterranean Diet and C677T MTHFR gene polymorphism interaction. Eur Rev Med Pharmacol Sci. 2013; 17(19):2555-65.

18. Frisard MI, Greenway FL, Delany JP. Comparison of methods to assess body composition changes during a period of weight loss. Obes Res. 2005; 13(5):845-54.

19. Bosy-Westphal A, Schautz B, Later W, Kehayias JJ, Gallagher D, Müller MJ. What makes a BIA equation unique? Validity of eight-electrode multifrequency BIA to estimate body composition in a healthy adult population. Eur J Clin Nutr. 2013; 67(Suppl 1):S14-21. doi: 10.1038/ejcn.2012.160

20. Bosaeus M, Karlsson T, Holmäng A, Ellegård L. Accuracy of quantitative magnetic resonance and eight-electrode bioelectrical impedance analysis in normal weight and obese women. Clin Nutr. 2014; 33(3):417-77. doi: 10.1016/j.clnu.2013.06.017

21. Riebl SK, Davy BM. The Hydration Equation: Update on water balance and cognitive performance. ACSM'S Health \& Fitness J. 2013; 17(6):21-8. doi: 10.1249/FIT.0b013e3182a9570f

22. Heyward VH, STOLARCZYK LM. Avaliação da composição corporal aplicada. São Paulo: Barueri; 2000.

23. Sillanpää E1, Cheng S, Häkkinen K, Finni T, Walker S, Pesola A, et al. Body composition in 18- to 88year-old adults-comparison of multifrequency bioimpedance and dual-energy $\mathrm{X}$-ray absorptiometry. Obesity. 2014; 22(1):101-9. doi: 10.1002/oby.20 583

24. Thomson R, Brinkworth GD, Buckley JD, Noakes $M$, Clifton PM. Good agreement between bioelectrical impedance and dual-energy X-ray absorptiometry for estimating changes in body composition during weight loss in overweight young women. Clin Nutr. 2007; 26(6):771-7.

25. Andreoli A, Melchiorri G, De Lorenzo A, Caruso I, Sinibaldi Salimei P, Guerrisi M. Bioelectrical impedance measures in different position and vs 
Dual-Energy X-ray Absorptiometry (DXA). J Sports Med Phys Fitness. 2002; 42(2):186-9.

26. Pateyjohns IR, Brinkworth GD, Buckley JD, Noakes M, Clifton PM. Comparison of three bioelectrical impedance methods with DXA in overweight and obese men. Obesity. 2006; 14(11):2064-70.

27. Lafortuna CL, Maffiuletti NA, Agosti F, Sartorio A. Gender variations of body composition, muscle strength and power output in morbid obesity. Int J Obes Relat Metab Disord. 2005; 29(7):833-41.

28. Regitz-Zagrosek V, Lehmkuhl E, Weickert M. Gender differences in the metabolic syndrome and their role for cardiovascular disease. Clin Res Cardiol. 2006; 95(3):136-47.

29. Coppini LZ, Waitzberg DL, Campos ACL. Limitations and validation of bioelectrical impedace analysis in morbidly obese patients. Curr Opin Clin Nutr Metab Care. 2005; 8(3):329-32.

30. Pérez-Matute P, Pérez-Martínez L, Blanco JR, Ibarra $V$, Metola L, Sanz M, et al. Multiple frequency bioimpedance is an adequate tool to assess total and regional fat mass in HIV-positive patients but not to diagnose HIV-associated lipoatrophy: A pilot study. J Int AIDS Soc. 2013; 16(1):18609.

31. Foss ML, Keteyian SJ. Bases fisiológicas do exercício e do esporte. Rio de Janeiro: Guanabara Koogan; 2000.

32. Wagner DR, Heyward VH. Techniques of body composition assessment: A review of laboratory and field methods. Res Q Exerc Sport. 1999; 70(2):135-9.

33. Hind K, Oldroyd B, Truscott JG. In vivo precision of the GE Lunar iDXA densitometer for the measurement of total body composition and fat distribution in adults. Eur J Clin Nutr. 2011; 65(1):140-2.

34. Silver HJ, Welch EB, Avison MJ, Niswender KD. Imaging body composition in obesity and weight loss: challenges and opportunities. Diabetes Metab Syndr Obes. 2010; 28; 3(1):337-47. doi: 10.2147/ DMSOTT.S9454

35. Kamel EG, McNeill G, Van Wijk MC. Usefulness of anthropometry and DXA in predicting intraabdominal fat in obese men and women. Obes Res. 2000; 8(1):36-42.

36. Bertin E, Marcus C, Ruiz JC, Eschard JP, Leutenegger M. Measurement of visceral adipose tissue by DXA combined with anthropometry in obese humans. Int J Obes Relat Metab Disord. 2000; 24(3):263-70.

37. Direk K, Cecelja M, Astle W, Chowienczyk P, Spector TD, Falchi $M$, et al. The relationship between DXAbased and anthropometric measures of visceral fat and morbidity in women. BMC Cardiovasc Disord. 2013; 13(1):25.
38. Rothney MP, Xia Y, Wacker WK, Martin FP, Beaumont $M$, Rezzi $S$, et al. Precision of a new tool to measure Visceral Adipose Tissue (VAT) using Dual-Energy X-Ray Absorptiometry (DXA). Obesity. 2013; 21(3):E134-6.

39. Hendel HW, Gotfredsen A, Andersen T, Hojgaard L, Hilsted J. Body composition during weight loss in obese patients estimated by dual energy X-ray absorptiometry and by total body potassium. Int J Obes Relat Metab Disord. 1996; 20(12):1111-9.

40. Toombs RJ, Ducher G, Shepherd JA, De Souza MJ. The Impact of Recent Technological Advances on the Trueness and Precision of DXA to Assess Body Composition. Obesity. 2012; 20(1):30-9.

41. IAEA Library Cataloguing in Publication Data Dual energy $X$ ray absorptiometry for bone mineral density and body composition assessment. Vienna: International Atomic Energy Agency; 2010.

42. McCrory MA, Gomez TD, Bernauer EM, Molé PA. Evaluation of a new air displacement plethysmograph for measuring human body composition. Med Sci Sports Exerc. 1995; 27(12):1686-91.

43. Waki M, Kral JG, Mazariegos M, Wang J, Pierson Jr RN, Heymsfield SB. Relative expansion of extracellular fluid in obese vs. nonobese women. Am J Physiol. 1991; 261(1):199-203.

44. Minderico CS, Silva AM, Teixeira PJ, Sardinha LB, Hull HR, Fields DA. Validity of air-displacement plethysmography in the assessment of body composition changes in a 16-month weight loss program. Nutr Metab. 2006; 3(1):32-40.

45. Vescovi JD, Zimmerman SL, Miller WC, Hilderbrandt L, Hammer I, Fernall B. Evaluation of the BODPOD for estimating percentage body fat in a heterogeneous group of adult humans. Eur J Appl Physiol. 2001; 85(3-4):326-32.

46. Le Carvennec M, Fagour C, Adenis-Lamarre E, Perlemoine C, Gin H, Rigalleau V. Body composition of obese subjects by air displacement plethysmography: The influence of hydration. Obesity. 2007; 15(1):78-84.

47. Weyers AM, Mazzetti SA, Love DM, Gomez AL, Kraemer WJ, Volek JS. Comparison of methods for assessing body composition changes during weight loss. Med Sci Sports Exerc. 2002; 34(3):497-502.

48. Hames KC, Anthony SJ, Thornton JC, Gallagher D, Goodpaster BH. Body composition analysis by air displacement plethysmography in normal weight to extremely obese adults. Obesity. 2013; 22(4):1078-84.

49. Lukaski HC. Methods for the assessment of human body composition: Traditional and new. Am J Clin Nutr. 1987; 46(4):537-56. 
50. Heath EM, Adams TD, Daines MM, Hunt SC. Bioelectric impedance and hydrostatic weighing with and without head submersion in persons who are morbidly obese. J Am Diet Assoc. 1998; 98(8):869-75.

51. Ellis KJ. Human body composition: In vivo methods. Physiol Rev. 2000; 80(2):649-80.

52. Mcardle WD, Katch FI, Katch VL. Fisiologia do exercício: energia, nutrição e desempenho humano. $4^{\mathrm{a}}$ ed. Rio de Janeiro: Guanabara Koogan; 1998.

53. Gallagher D, Thornton JC, He Q, Wang J, Yu W, Bradstreet $T E$, et al. Quantitative magnetic resonance fat measurements in humans correlate with established methods but are biased. Obesity. 2010; 18(10):2047-54.

54. Lee S, Kuk JL. Changes in fat and skeletal muscle with exercise training in obese adolescents: Comparison of whole-body MRI and dual energy X-ray absorptiometry. Obesity. 2013; 21(10):2063-71.

55. Brooke-Wavell K, Jones PR, Norgan NG, Hardman $A E$. Evaluation of near infra-red interactance for assessment of subcutaneous and total body fat. Eur J Clin Nutr. 1995; 49(1):57-65.

56. Panotopoulos G, Ruiz JC, Guy-Grand B, Basdevant A. Dual x-ray absorptiometry, bioelectrical impedance, and near infrared interactance in obese women. Med Sci Sports Exerc. 2001; 33(4):665-70.

57. Prado CM, Bekaii-Saab T, Doyle LA, Shrestha S, Ghosh S, Baracos VE, et al. Skeletal muscle anabolism is a side effect of therapy with the, MEK inhibitor: Selumetinib in patients with cholangiocarcinoma. $\mathrm{Br} J$ Cancer. 2012; 106(10):1583-6. doi: 10.1038/bjc.2012.144

58. Pontiroli AE, Pizzocri P, Giacomelli M, Marchi M, Vedani $P$, Cucchi $E$, et al. Ultrasound measurement of visceral and subcutaneous fat in morbidly obese patients before and after laparoscopic adjustable gastric banding: Comparison with computerized tomography and with anthropometric measurements. Obes Surg. 2002; 12(5):648-51.

59. Radominski RB, Vezozzo DP, Cerri GG, Halpern A. O Uso da ultra-sonografia na avaliação da distribuição de gordura abdominal. Arq Bras Endocrinol Metab. 2000; 44(1):5-12.

60. Pineau JC, Lalys L, Bocquet M, Guihard-Costa AM, Polak M, Frelut ML, et al. Ultrasound measurement of total body fat in obese adolescents. Ann Nutr Metab. 2010; 56(1):36-44.

61. Park BS, Yoon JS. Relative skeletal muscle mass is associated with development of metabolic syndrome. Diabetes Metab J. 2013; 37(6):458-64. doi: 10.4093/dmj.2013.37.6.458

62. Baumgartner RN, Heymsfield SB, Lichtman S, Wang J, Pierson RN Jr. Body composition in elderly people:
Effect of criterion estimates on predictive equations. Am J Clin Nutr. 1991; 53(6):1345-53.

63. Heymsfield SB, Wang J, Aulet M, Kehayias J, Lichtman S, Kamen $Y$, et al. Dual photon absorptiometry: validation of mineral and fat measurements. In: Yasumura S, Harrison JE, McNeill $K G$, Woodhead $A D$, Dilmanian FA, Editor. In vivo body composition studies. New York: Plenum Press; 1990.

64. Baumgartner RN, Koehler KM, Gallagher D, Romero L, Heymsfield SB, Ross RR, et al. Epidemiology of sarcopenia among the elderly in New Mexico. Am J Epidemiol. 1998; 147(8):755-63.

65. Janssen I, Heymsfield SB, Baumgartner RN, Ross R. Estimation of skeletal muscle mass by bioelectrical impedance analysis. J Appl Physiol. 2000; 89(2):465-71.

66. Cruz-Jentoft AJ, Baeyens JP, Bauer JM, Boirie Y, Cederholm T, Landi $F$, et al. European Working Group on Sarcopenia in Older People. Sarcopenia: European consensus on definition and diagnosis: Report of the European Working Group on Sarcopenia in Older People. Age Ageing. 2010; 39(4):412-23. doi: 10.1093/ageing/afq034

67. Davison KK, Ford ES, Cogswell ME, Dietz WH. Percentage of body fat and body mass index are associated with mobility limitations in people aged 70 and older from NHANES III. J Am Geriatr Soc. 2002; 50(11):1802-9.

68. Ritz P. Obesity in the elderly: Should we be using new diagnostic criteria? J Nutr Health Aging. 2009; 13(3):168-9.

69. Batsis JA, Barre LK, Mackenzie TA, Pratt SI, LopezJimenez F, Bartels SJ. Variation in the prevalence of sarcopenia and sarcopenic obesity in older adults associated with different research definitions: Dualenergy X-ray absorptiometry data from the National Health and Nutrition Examination Survey 1999-2004. J Am Geriatr Soc. 2013; 61(6):974-80.

70. Coxam V. [New advances in osteoporosis nutritional prevention]. Med Sci (Paris). 2005; 21(3):297-301.

71. Looker AC, Flegal KM, Melton LJ. Impact of increased overweight on the projected prevalence of osteoporosis in older women. Osteoporos Int. 2007; 18(3):307-13.

72. Castro JP, Joseph LA, Shin JJ, Arora SK, Nicasio J, Shatzkes J, et al. Differential effect of obesity on bone mineral density in White, Hispanic and African American women: A cross sectional study. Nutr Metab (Lond). 2005; 2(1):9.

73. Beck TJ, Petit MA, Wu G, LeBoff MS, Cauley JA, Chen Z. Does Obesity Really Make the Femur Stronger? BMD, Geometry, and Fracture Incidence in the Women's Health Initiative-Observational 
Study. J Bone Miner Res. 2009; 24(8):1369-79. doi: 10.1359/jbmr.090307

74. World Health Organization. Assessment of fracture risk and its application to screening for postmenopausal osteoporosis. Géneve: WHO; 1994. Technical Report Series, nº 843.

75. Petak S, Barbu CG, Yu EW, Fielding R, Mulligan K, Sabowitz $B$, et al. The official positions of the international society for clinical densitometry: Body composition analysis reporting. J Clin Densitom. 2013; 16(4):508-19. doi: 10.1016/j.jocd.2013. 08.018

76. Marwaha RK, Garg MK, Tandon N, Mehan N, Sastry A, Bhadra K. Relationship of body fat and its distribution with bone mineral density in Indian population. J Clin Densitom. 2013; 16(3):353-9. doi: 10.1016/j.jocd.2012.08.074

77. National Institutes of Health Consensus Development Conference Statement. Health implications of obesity. Ann Intern Med. 1985; 103:147-51.

78. Gudbergsen H, Lohmander LS, Jones G, Christensen $R$, Bartels EM, Danneskiold-Samsoe B, et al. Correlations between radiographic assessments and MRI features of knee osteoarthritis: A crosssectional study. Osteoarthritis Cartilage. 2013; 21(4):535-43. doi: 10.1016/j.joca.2012.12.010
79. Zemel BS. Quantitative computed tomography and computed tomography in children. Curr Osteoporos Rep. 2011; 9(4):284-90. doi: 10.1007/s11914-011-0 076-x

80. llich JZ, Kelly OJ, Inglis JE, Panton LB, Duque G, Ormsbee MJ. Interrelationship among muscle, fat, and bone: Connecting the dots on cellular, hormonal, and whole body levels. Ageing Res Rev. 2014; 15:51-60. doi: 10.1016/j.arr.2014.02.007

81. Ormsbee MJ, Prado CM, llich JZ, Purcell S, Siervo M, Folsom A, et al. Osteosarcopenic obesity: The role of bone, muscle and fat on health. J Cachexia Sarcopenia Muscle. 2014; 5(3):183-92.

82. Waters DL, Hale L, Grant AM, Herbison P, Goulding A. Osteoporosis and gait and balance disturbances in older sarcopenic obese New Zealanders. Osteoporos Int. 2010; 21(2):351-7. doi: 10.1007/s00 198-009-0947-5

83. Pasco JA, Gould H, Brennan SL, Nicholson GC, Kotowicz MA. Musculoskeletal deterioration in men accompanies increases in body fat. Obesity. 2014; 22(3):863-7. doi: 10.1002/oby.20496

Recebido em: 6/4/2014 Aprovado em: 4/9/2014 
